\title{
Electrochemical metamaterials
}

\section{Alexei A. Kornyshev ${ }^{1}$}

Received: 8 July 2020 / Revised: 8 July 2020 / Accepted: 8 July 2020 / Published online: 25 August 2020

(C) The Author(s) 2020

The role of electrochemistry in energy systems such as batteries, fuel cells, capacitors, solar cells and water desalination devices is commonly known to wide public. Many research groups worldwide are involved in a race to make those electrochemically based technologies more efficient, economically viable and environmentally friendly. A lot of progress has already been made, for example fully electric or hybrid cars are seen on our streets, experimental fuel cell buses, supercap-driven trams being recharged at each stop, construction cranes recuperating energy by charging supercaps when laying down their platforms before loading, solar panels in private households, installations for the production of fresh water from sea brine or even from human urine at the international space station - all became reality. For electrochemists, being involved in any part of that research and development make them feel good and needed, contributing to the fight against global warming and making impact to our way of life. Last but not least, it is the safest way to get their research funded.

Much less widely understood is the key research in such areas as electrochemical kinetics and electrocatalysis, underpinning most of those mentioned technologies as well as metal treatment, corrosion science, production of gases by electrolysis, electrochemical synthesis, sensors and many other classical 'mainstream' branches of applied electrochemistry.

Generally, the societal priorities of the XXI century in its 3rd decade and expectedly in the next few ones, which determine the priority areas for research funding, can be summarized as shown in a diagram:

Alexei A. Kornyshev

a.kornyshev@imperial.ac.uk

1 Department of Chemistry, Imperial College London, Molecular Sciences Research Hub, White City Campus, London W12 0BZ, and Thomas Young Centre for Theory and Simulation of Materials, Imperial College London, South Kensington Campus, London SW7 2AZ, UK

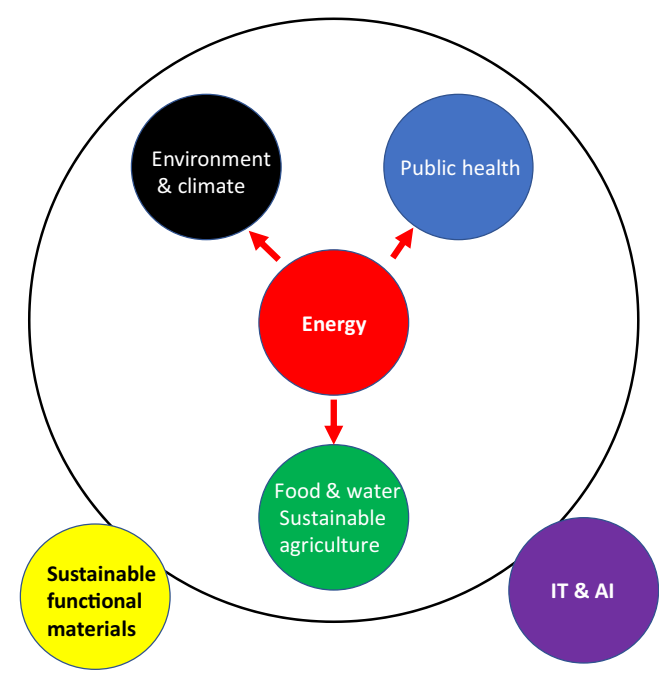

Advanced and sustainable manufacturing is not depicted here, as implicit. All of these subjects are deeply inter-related.

Of course, nothing can be done without energy. Public health and well-being are affected by climate, by supply of food and water, which in turn affect and are affected by climate. The whole circle and development of the healthy relationship between its components are boosted by information technologies and artificial intelligence, as well as progress in new materials (although both, as we know, as well as other elements of this scheme if misused, may contribute to 'pollution').

As described above, in 'Energy' the role of electrochemistry is more or less obvious, and it, of course, contributes to "Climate" through the production of clean energy. There are also other inputs to its related tasks, in the first place through capture or consumption of carbon dioxide, production of hydrogen as a fuel for hydrogen economy via electrolysis or photo-electrolysis, ultra-filtration and treatment of exhaust water, and of course the issues related to 'Energy' enriched with such new directions, as 'blue energy'. In 'Public health', the input is less straightforward, underpinned by biolectrochemistry (protein functioning, biological membranes, nerve signal propagation, pace makers) or molecular electrochemistry for sensing. Achievements in 'Information Technologies and Artificial Intelligence' are rather used in electrochemistry, than benefiting 
from the latter. In 'Food et al.', this could be, e.g. electrochemical synthesis of ammonia for fertilisers avoiding the production of $\mathrm{CO}_{2}$ as a side product, and of course the mentioned desalination.

Electrochemistry, indeed, seems to be one of the 'cleanest' sciences; it is appropriate to cite here Frumkin's metaphor from his unforgettable Mendeleev lecture in Alma Aata 50 years ago: 'Aphrodite came out of sea froth (concentrated electrolyte solution), but not from oil'.

As for 'Materials', apart from classical electrochemical synthesis and catalysis of various substances, it is a relatively fresh avenue, and in this short essay, I want to focus on it. Moreover, I will dwell on its least advanced but perhaps hottest direction-design of electrotunable metamaterials.

What are metamaterials? These are materials with unusual, odd, disruptive, funky, 'crazy', fancy (you can continue yourself the list of such adjectives) properties. Not all of the discovered and designed metamaterials, may appear useful in the end. But some of them do or at least promise to open entirely novel routs for providing new functions. For me, the best symbol for such materials is as shown below, taken from the world of modern architecture.

This picture symbolises for me the pros and contras of this field. Indeed, it demonstrates the unlimited fantasy of the architect. Its shape puzzles spectators, their-“wow!" guaranteed. But, (i) it is a few times more expensive than a conventional building of that size; (ii) the judgement on its functionality can be a matter of taste, and, sadly, (iii) such buildings often get tired faster than 'classical' buildings (see the spectacular Gehry's constructions at MIT campus in Cambridge MA; that equally refers to some 'meta-buildings' of his passed away 'sister-in-arms', brilliant Zaha Hadid).

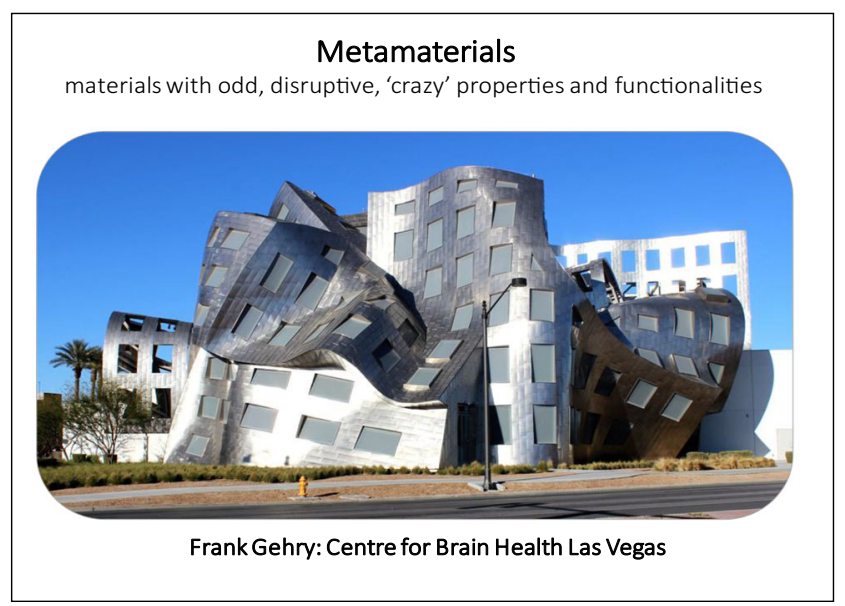

The same story is with metamaterials. Not all of them will be able to compete with the conventional ones, some may degrade faster; and will they be cheap enough to create and use them? Still, you cannot stop the human imagination, and this area is extremely attractive for its novelty and the brilliance of ideas advancing it.
The term metamaterials is, however, almost a synonym of optical metamaterials, i.e. those that deliver new optical properties. These are, e.g. quantum metamaterials, nonlinear and amplifying metamaterials, materials for transformation optics, invisible cloaks, designer dispersions for slowing light, chiral metamaterials, sensor metamaterials (e.g. based on SERS), microwave frequency selective materials, all kind of complex optical filters and light trappers [1]. All of these are the key components of what nowadays is called photonics $[2,3]$, the term widely used to embrace the directions of modern optics that use metamaterials or tricky electrodynamic phenomena to produce novel optical effects.

The materials that deliver interesting mechanical properties and their related exploitation are usually called functional materials $[4,5]$; although this term may be questioned, at least I do not like it: what is the point for us to work on and develop materials which are not functional? Except, maybe, for the case when they are purely structural, but even then, they have their clear function! Instead of functional, the term smart materials is widely used [6], underlining the idea that they can do something that other, 'less-smart' materials cannot (or that they are designed by smart people?).

One class of such materials which is particularly smart are polymer electrolyte electroactuators for robotics [7], reverse actuators such as harvesters of electricity from walking or any other mechanical motion (see e.g. references on piezo $[8,9]$, capacitive [10-12] and nanotribological [13, 14] devices, and the literature cited there). To avoid ambiguity, I prefer to call all those materials - mechanical metamaterials. Many of them related with effects that are underpinned by electrochemistry. These materials and physical phenomena they are based upon will require a special coverage. Thus, in these notes, I will concentrate on the perspectives of electrochemistry for optical metamaterials.

The science and engineering of the latter have made great progress due to advances in both theory and in micro and nanoengineering. Theoretical analysis facilitated by rapidly developing novel computational tools resulted in suggestions of new, original scenarios and architectures of such materials for their experimental validation. Grand progress in nanotechnology in the last three decades, allowed for such materials to be constructed. But the majority of them had one common problem. Fabricated based on complex nanotechnology, they were static, performing fixed, given function(s). Already at META-2014 congress in Singapore, Nikolay Zheludev in his opening speech said: 'The time of metamaterials is...over. It is the time of tuneable metamaterials'. He and his group have been already working in this area, either coming up with original scenarios of controlling light with light [15] or deforming metamaterials mechanically [16]; there were also a number of other groups who had achieved tunability of optical meta-structures by mechanical stress [17-20]. 
Electrochemistry is, however, in the unique position here to offer new scenarios for electrotunability, and what is specific for electrochemistry - by tiny voltage variation. By this term, we mean voltage control of the properties of various selfassembling optical systems. The origin of this 'hope' goes back to old times.

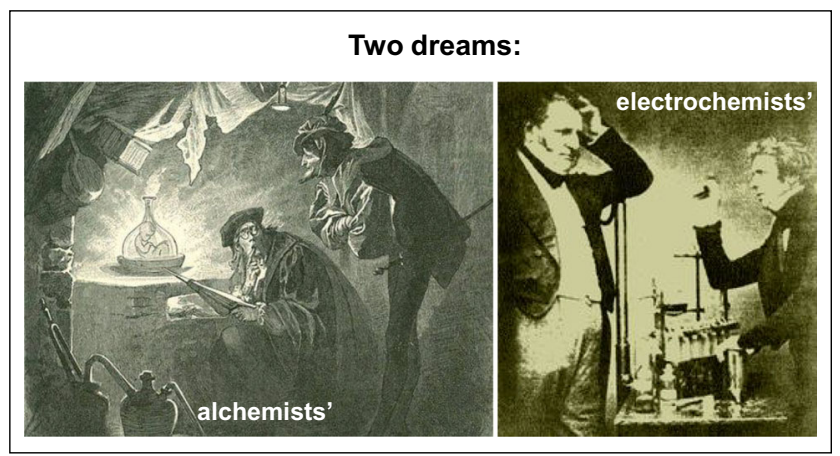

A dream of a medieval alchemist was to find a magic composition of a solution under which a homunculus will self-assemble in a test tube. A dream of an electrochemist, already since Faraday times, has been to apply and vary the voltage in an electrochemical cell, and whatever selfassembles or disassembles at the electrochemical interface will depend on the electrode potential. Paraphrasing Dostoyevsky, who said that 'Beauty will save the World', a maverick electrochemist would say the same about the potentiostat.

The first of the two dreams has not been realised literally, homunculus-wise, but after many circles has brought us to modern supramolecular chemistry, polymer chemistry, and colloid science, and it neither escaped the attention of electrochemists [21]. The realisation of the second dream is a continuous process.

We will speak below about the use of both approaches to control first of all the properties of plasmonic metamaterials [22]. But before describing that step-few words on plasmonics. This is a branch of solid-state physics and optics exploring different effects of collective electron plasma excitations (plasmons) in the optical response of interfaces and interfacial films, and exploiting them in various optical, sensing and catalysing processes [23]. Plasmonics took off in the $1960 \mathrm{~s}-1970 \mathrm{~s}$, with the pioneering works of Otto [24] and Kretschmar [25, 26] who formulated the principles of techniques for characterisation of surface plasmons and experimental measurements of the spectra of these collective excitations, whereas the concepts of bulk plasmons and detections of surface plasmons in thin films were proposed already in 1950s [27, 28].

Plasmonics entered electrochemistry and was actually boosted by electrochemistry, more than 40 years ago with the discovery of surface-enhanced Raman scattering (SERS) in the 1970s. The famous paper, from the University of
Southampton, by Fleischman, Hendra, and McQuillan [29], first reported on the effect of a 'giant' enhancement of Raman signal from pyridine molecules adsorbed on a rough silver electrode; but they did not unravel the nature of this effect. 'Purists' on the other side of the Atlantic used to say that the Southampton team has observed the effect, whereas Van Duyne [30] at North Western University, with his systematic studies, has unravelled what stands behind it. They attribute the discovery of SERS rather to him, and (justly) acknowledge those who contributed to its scientific understanding (see pioneering theoretical works of Schatz, Moskowitz, and others, as reviewed in [31-33]). Indeed, it had slowly become clear that the giant enhancement of Raman signal from molecules adsorbed on rough surfaces is in the first place related to plasmon resonance due to a possibility of direct excitation of surface plasmons by light incident on a rough surface. First estimates, confirmed later with the development of computational methods of solving Maxwell equations, made it clear that local values of electric component of electromagnetic radiation, $E$, with frequencies close to surface plasmon ones, maybe resonance-enhanced near the tips and in crevices of the rough metal surface by a couple of orders of magnitude. Since the intensity of Raman signal from the analyte molecule scales roughly as $E^{4}$, one can end up with some $10^{8}$ times enhanced signals. Although, other factors responsible for the enhancement of the Raman signal (the so-called 'chemical' or charge transfer enhancement) have been added to the picture beginning with the paper by Albrecht and Creighton [34], and later dwelled upon by Otto [35], the plasmon-resonance mediated enhancement remains central for SERS. In 1980s, interest to plasmons in electrochemistry beyond SERS coincided with development of electromodulation spectroscopy $[36,37]$

The boom in nanotechnology and nanoscience of this century gave rise to the second birth of plasmonics, more generally photonics (the science that including plasmonics as a particular case). Indeed, conventional materials derive their optical properties from atoms and molecules. Nanoand micro-technology could create structures and architectures from larger blocks than simple atoms, e.g. nanoparticles or other components of nanostructures, thus designing larger scale 'atoms' to access new functionalities, capitalising on plasma excitations in these complicated structures or more complex effects [38].

Electrochemistry very often rapidly followed discoveries in physics, and there are many examples of it in the history of science. In recent times, some of those were building the in situ STM with atomic resolution which could picture atomistic structure of electrode/electrolyte interface [39, 40], just 2 years after Binning and Rohrer reported their vacuum STM [41, 42], or implementation in a due time of just discovered carbon nanotubes or graphene for building electrode for electrochemical supercapacitors [43]. Electrochemistry had not exploited 
the achievement of modern plasmonics in full yet but it can do it, by using nano-engineered electrodes [44-46] or playing with self-assembling nanostructures, and this is what is happening now.

The simplest of such self-assembling nanostructures were arrays of metallic nanoparticles (NPs) at ITIES. The road to these works was opened by pioneering papers of Schiffrin, Girault, Samec and their groups [47-51], who have studied the formation of such layers at electrochemical liquid|liquid interfaces (LLI) [52, 53], i.e. interfaces of immiscible electrolytic solutions (ITIES), including their optical and/or electrical (capacitance) characterisation. Indeed, at LLI properly functionalised NPs, usually dissolved in the aqueous phase tend to spontaneously adsorb onto the interface to block the unfavourable contact between water and oil. Voltage control over electrosorption of NPs was demonstrated (they were, however, too small, of $1.5 \mathrm{~nm}$ in diameter, for any contribution to modification of the linear optical signal) already in Ref. [51] through cyclic voltammetry and capacitance measurements. Second-harmonic generation detecting the presence of NP was first demonstrated in Ref. [50], but again for smallish NPs, $6 \mathrm{~nm}$ in diameter.

In 2010, Flatte, Urbakh and I proposed and explored an idea how to influence optical properties of ITIES through voltage controlled assembly disassembly of NP arrays, by tuning concentration of electrolyte and potential drop across the interface [22]. With all the preliminary character of the estimates presented in that Feature Article and some minor inaccuracies, this theoretical paper has set for us a new direction of research: electrotuneable electrochemical plasmonics.

Indeed, the quasi-2d NP arrays of plasmonic MPs (gold, silver, titanium nitride, core-shell composite NPs) adsorbed at the interface of two optically transparent media reflect light in a broad visible range with a maximum reflection centred about the frequency of the localised plasmon modes in nanoparticles. That value depends on the size and composition of NPs; for $20 \mathrm{~nm}$ diameter AuNP, it is close to the wavelength of $560-580 \mathrm{~nm}$. The reflection signal depends on the material and size of NPs: the reflection signal is weaker the smaller the size and the stronger is light absorption in the material. Too large sizes of NPs, approaching the wavelength of light, would neither be good for reflection, as this would give rise to diffuse scattering of light. The size between 16 and $40 \mathrm{~nm}$ in diameter was found to be most suitable for enhanced reflection. Apart from the size, another critical parameter is the average distance between NPs in the arrays, i.e. the array density. It affects the reflection spectra in two ways: (i) the reflection at maximum and across its whole spectrum increases, the closer to each other are the NPs in the array; (ii) the wavelength of maximum is shifted to the red with increasing the array density.
Thus, the following picture and manipulation scheme was envisaged and later approved by a series of systematic experimental studies:

1. NPs are functionalized with ligands, such as mercaptanoic acid or citrates. Having acidic terminal groups, they dissociate in water, retaining negative charges (other ligands may be designed to deliver a positive charge, but the sign of the charge is not principle rather its value, as long as it is the same for all NPs). The charge thus associated with NPs will protect them against agglomeration that may be caused by van der Waals forces (a standard measure for stabilisation of colloids). A $20 \mathrm{~nm}$ NP functionalized at neutral $\mathrm{pH}$ by mercapatanoic ligands can bear approximately 900 elementary charges.

2. The electrostatic repulsion of charged NPs can be controlled through the variation of electrolyte concentration that affects Debye screening and through the degree of ionisation of ligands, which can be altered by the variation of $\mathrm{pH}$. More concentrated electrolytes will stronger screen the electrostatic repulsion in the solution. More acidic $\mathrm{pH}$ will reduce the degree of dissociation of acidic terminal groups of functionalised ligands and decrease the overall charge associated with NPs. Both factors will reduce the repulsion of NPs in the array adsorbed at the interface and would favour denser arrays. But obviously, this cannot be overdone - too short screening length in the solutions and too small charges of NPs would lead to their agglomeration in the bulk.

3. The control of inter-NP spacing through electrolyte concentration and $\mathrm{pH}$ is, thus, limited. although each individual NP would wish to settle at the LLI, as we have designed the system in such a way that NPs repel each other strong enough not to fuse with each other in the bulk, the NPs will keep 'social distance' between each other when adsorbed at the interface. Thus, the strategy is to reduce repulsion as much as possible, but not that much that they would start agglomerating in the bulk (and then at the interface). Theory may try to estimate the limiting values for electrolyte concentration and $\mathrm{pH}$, but they are best defined for each system experimentally as has been repeatedly done. Indeed, agglomeration of NPs in the bulk can easily be detected through the characteristic features in the light extinction spectra of the solution bulk. All in all, each such system will have some minimal distance for interparticle separation in an adsorbed array. Since nanoparticles were designed to repel each other, for spherical NPs that array will be, on average, hexagonal.

4. If we want NPs to form even denser arrays, a natural idea would be to apply voltage across the ITIES to push the charged NPs to the interface. If the ligands are negatively charged, this will take place polarising aqueous phase negatively. When positively charged ligands are used, this 
will be other way around. Polarising the cell correspondingly will increase the driving force for NPs to settle at the interface. The capillary well and solvation does not let them go into the oil phase completely, so that they settle at the ITIES, piercing it, although when being pushed by electric field they can be somewhat shifted to the oil side of the interface. This would make the potential well for each individual NP deeper, and thereby increase their 'desire to settle at the interface', so that NPs will tolerate shorter social distancing between each other.

These ideas were touched in different depths in the 2010 paper, but all their details and complications to overcome were made clear only after a series of follow up theoretical works and brand new experimental investigations, performed for both for LLI and solid/liquid interfaces (SLI).

The theoretical works focussed on the development of a more accurate theory of optical signals from NP arrays settling at optically transparent as well as reflective interfaces, at a later stage tested by full-wave simulations of optical signals. The theory was reduced to precision by Debabrata Sikdar leading to excellent correspondence between theory and fullwave simulations $[54,55]$. Systematic realisation of these principles took place at Imperial College, in the laboratory of Joshua Edel-led by him, Anthony Kucernak, and myself, with a crucial work of research associates-Eleanora Velleman, Yunuen Montelongo, and Ye Ma, and few other students and co-workers. This has not only built an experimental basis of electrotuneable electrochemical plasmonics, but also allowed to systematically test the theory on various optical experimental data. I will mention just a few milestones of that research programme.

That period of work was actually preceded by a series of earlier works, performed before achieving electrovariability experimentally, and reviewed in Ref. [56] It has started with experimental investigation of the factors influencing adsorption of NPs onto LLI (electrolyte concentration and the aqueous solution $\mathrm{pH}$ ) [57] followed by computational analysis of the optical properties of NPs at the interface [58]. The 'nonelectrotunable' period culminated in Ref. [59], which has demonstrated an unprecedented level of detection of Raman signals from analyte molecules adsorbed between 40-nm-sized NPs adsorbed at LLI. Again, in that work, control of the density of the adsorbed NP array was only via variation of electrolyte concentration and $\mathrm{pH}$. But it has shown how 'hot' can be the spots of enhancement of electromagnetic radiation (near and in between the NPs) probing the vibrational modes of the analyte molecules through their 'Raman fingerprints'. Although that work did not involve yet an electrochemical setup, and thus no voltage could be applied there to stabilise and densify the adsorbed NP arrays, it was unambiguous evidence of the formation of relatively dense NP arrays at an LLI.
There was then a seminal paper published by the group led by Mark Schlossman at the University of Illinois in Chicago. They have performed very fine investigations of assembly of small ( $2 \mathrm{~nm}$ ) NPs functionalized by positively charged ligands [60]. Their localization at the water/DCE ITIES was studied by Grazing Incidence Small Angle Scattering (GISAXS), controlled by the voltage applied across the interface. From Bragg diffraction peaks, they could see a hexagonal arrangement of NPs at the interface, with the decrease of the lattice constant, when NPs are get pushed towards oil by electric field at the interface. Through X-ray reflectivity, they could also assess how deep the NPs can be pushed towards oil, when polarising water positively with respect to oil. The molecular dynamic simulations performed in the same work indicated that when NPs are pressed into the oil side of the interface, their charge seems to be strongly compensate by the counterions binding to the charged groups of the functionalizing ligands.

That work has inspired Joshua Edel to initiate and lead a study, crucial for the whole following work of the Imperial team in this area. The results of this study were published in Ref. [61], which referred to a detailed structural and optical characterisation of NP arrays at the LLI. It was the measurements of spectra of light reflection from NP arrays spontaneously adsorbed at LLI, the density of which was controlled by variation of electrolyte concentration either in aqueous or in organic phase, with simultaneous characterisation of the array structure by X-ray diffraction. The experiments were performed at the Dimond Synchrotron Light Source at Harwell. Importantly, the X-ray diffraction and light reflection measurements were performed at the same time in the same cell. Again, it was not an electrochemical cell, and the light reflection was not strong, because too dense arrays did not form without assistance of applied voltage, and furthermore the used gold NPs were of intermediate size, $12.8 \mathrm{~nm}$. But the reflection spectra, performed at normal incidence of light to the LLI, were still well detectable. The X-ray diffraction data has shown almost ideal hexagonal structure of the arrays and the expected decrease of the inter-NP distance with the increase of electrolyte concentration. That distance was a key parameter of the theory of light reflection from the array. Thus, for each electrolyte concentration, and thereby interNP separation in the array, determined experimentally, it was possible to calculate practically without adjustable parameters the whole reflection spectra and compare them with the experimentally measured ones. The results have shown excellent correspondence of theory [54] and experiments, thus proving that the basic ideas underpinning the whole project were correct.

Let us reiterate that the size of NPs is crucial for the mirror effect. The smaller the NP, the smaller the reflectivity at maximum, and the intensity goes down overall spectrum correspondingly. Girault's group [62] has studied systematically the size effect, controlling the population of LLI with NPs of 
different size by direct addition of NPs to the interface (the method not excluding formation of multilayers of NPs). It was found, not unexpectedly, that already above $60 \mathrm{~nm}$ in diameter of NPs, the scattering effects seemingly start to interfere with reflection, as reflection goes down with further increase of the size of NPs (see their Fig. 4). There is thus an optimal NP-size for an 'ideal' mirror, as the wave-length must be much larger than the diameter of NPs, to see it as a smooth surface. At the same time, if the NPs are too small, its 'plasmonic mass' will also be too small, and the presence of such NPs at the interface will be invisible in linear optics. Interestingly, in this work, the population of the interface was controlled also by measuring the electronic conductivity across the interface (electrons presumably tunnelling along the ligands between the NPs along their quasi-2d percolation network). So, in Ref. [62], they also plotted the reflection signal as a function of estimated NPpopulation at the interface.

As mentioned, the experiments at Diamond did not and could not yet involve any elements of electrovariability. It thus remained to demonstrate the latter in an ITIES electrochemical cell. The corresponding opto-electrochemical setup has been built under supervision of Edel and Kucernak. The experiments where performed with $16 \mathrm{~nm}$ AuNPs adsorbing at/desorbing from aqueous/1.2-DCE interface at optimised $\mathrm{pH}$ of the aqueous phase and concentrations of $\mathrm{Na}^{+} \mathrm{CL}^{-}$electrolyte in it, and $\mathrm{TBA}^{+} \mathrm{TPB}^{-}$in organic phase. The experimentally measured reflection spectra at normal incidence, obtained for each given voltage across the ITIES, where treated with the theory by fitting first the wavelength of reflection maxima and their height, thereby extracting the information on the average inter-NP separation (as the were no independent X-ray data available for it), and then calculating the whole spectra. It was full success again: the calculated spectra where practically exactly like the measured ones. This was the first realisation and characterisation of electrovariable mirror at an electrochemical interface [63].

Joshua Edel has shot a video, showing how this mirror looks like, and how it operates: https://www.nature.com/ nmat/journal/vaop/ncurrent/full/nmat4969.html The video has been viewed on the web more than 24,000 times, and discussed on many websites, see, e.g. https://www. photonics.com/Articles/Tunable_Nanoparticle_Layer_ Switches_Between/a62514.

It shows how a tiny, just $0.5 \mathrm{~V}$, variation of voltage can change the interface from a 'mirror' to 'window' mode. It displays either the reflection of a coin positioned above the interface onto which the light is shined when AuNp form a dense array. When the voltage is switched to push NPs away from the interfaces to the bulk of the aqueous phase, thereby making the interface transparent, you see a $£ 10$ banknote underneath.

The video, as spectacular as it is, had, however, to be dramatically sped up, because those experiments deliberately avoided dielectrophoresis $[64,65]$ that could otherwise drive charged NPs to the interface through the aqueous bulk. The static applied voltage cannot do it. In the absence of electrical current across the interface electric fields in the bulk of each phase are screened and both aqueous and oil bulks stay electroneutral. The electric field is concentrated only within the electrical double layers on either side of the interface and at the electrodes. Thus, when the aqueous phase was polarised more negatively, increasing thereby the potential wells that trap NPs at the interface, NPs in the bulk could not know anything about it. They would feel the difference, only when they get close to the LLI. But they could reach the interface to get trapped there only via random diffusion from the bulk, which is a slow process. Compare yourself reaching the point of interest in a historical town with a guide or by random walk.

A simple estimate shows that the diffusion time for NPs to reach the interface to form a monolayer is inversely proportional to the square of the bulk concentration, $c$, of NPs [63]. Increasing their concentration through the increase of the number of NPs would not, however, be a good idea, because the aqueous solution in which such NPs will be dissolved would get heavily coloured - it will look like Bordeaux wine. But one can reduce the thickness of the aqueous phase, maintaining a minimal number of NPs (just enough to cover the monolayer at the interface). This has been tested experimentally, and the $1 / c^{2}$ law for the switching time was approved [63]. This opens a door to building electrochemical microcells, without invoking dielectrophoresis (involvement of the latter is possible, but it would have made practical implementations of such cells more complicated). Estimates show that properly designed microcells could deliver millisecond switching time between window and mirror modes.

With a dream to use such optical switches in various applications, and particularly in the building industry - switching windows to mirrors in skyscrapers, saving energy on air conditioning when offices are not occupied, as first spelled out in Ref. [22], which may require vertical orientation of the interface-ITIES do not look too practical. Electrochemical cells with vertical ITIES have been created from the early days of ITIES in laboratory (see papers cited here), but no one tried to upscale them. So our thought was to create similar optical switches on the basis of adsorbing/desorbing NPs from electrolytes onto/from transparent electrodes, such as ITO, $\mathrm{ZnO}$, or graphene on a glass-substrate. Corresponding calculations have been performed showing very promising results. But this idea has not yet been experimentally realised. In the meantime, a more conventional (for electrochemistry) prototype of such system was considered.

Indeed, a purely theoretical analysis, confirmed also by full wave simulations, has shown another interesting effect, when NPs get electrosorbed or electrodesorbed on a mirror metal electrode [55]. Without NPs adsorbed on the surface of such electrodes, it should provide classical wide band reflection 
spectrum of that bare surface. But as predicted by the theory, with a dense monolayer of plasmonic NPs of sufficiently large size adsorbed on it, another effect should take place: a broadband quenching of reflection. The wavelength of the reflection dip is centred again at the wave-length of plasmon resonance of individual NPs, moving slightly to the red with densification of the array.

This effect was not broadly known. All aspects of it were clearly described in Ref. [55], but it was also predicted within an earlier version of the effective medium theory [66] (substantially improved in Ref. [55]), and by using another theoretical method by Truong and de Dormale [67]. In another configuration (adsorption of AuNP on thin gold film that allowed studying light reflection from the adsorbed NP-array side as well as shining light onto the other side of the film, thus probing plasmon resonances by evanescent wave excitation), an effect of similar nature was experimentally studied by the group of David Smith [68]. Moreover, in an overlooked early paper of the Liverpool group led by David Schiffrin signatures of a similar effect have been seemingly observed in the electroreflectance signal from the bi-layer of $5 \mathrm{~nm}$ AuNps on functionalized gold electrode [69].

Somehow, predictions of the theory [55] have been systematically tested experimentally by the Imperial team [70]. Silver electrode, protected by nano-thin layer of titanium nitride (thin enough not to affect optical properties of the silver surface) in aqueous solution of AuNPs functionalized by negatively charged ligands, was shown to experience the described quenching of reflection with electrosorption of NPs, returning the optical reflectivity of bare silver in water with their electrodesorption. Within the electrochemical window, adsorption of negatively charged NPs was stimulated by continuous variation of electrode potential from PZC to $+0.6 \mathrm{~V}$ relative to the latter: each electrode potential resulted in different density of the NP array, and correspondingly, the more positive the electrode potential resulted in denser arrays of adsorbed NPs and the stronger reflection quenching. Within the acquired potential window, the array was never denser than the one corresponding to $5 \mathrm{~nm}$ inter-NP surface-to-surface separation. But even then, as was found in a later paper [71], for large NPs $(40 \mathrm{~nm}$ in diameter), almost $100 \%$ quenching of reflectance was observed at the wavelength of reflection minimum. All the experimentally obtained spectra were fitted to the theory, through the wavelength of the reflection dip and its depth, with one fitting parameter - the average distance between NPs. Thus, the average inter-NP separation was retrieved for each applied electrode potential. Then, again, using the values of this key parameter of the theory, the whole spectra were calculated, reproducing amazingly well the experimental curves.

Encouraged by this success, the team went back to NParray-based SERS measurements, now not on LLI (work in progress), but on a metal substrate. Physically, the space between electrosorbed NPs (still protected against direct contact with the metal substrate by their functionalizing ligands) and the electrode deliver hot spots for electromagnetic radiation, even when the NPs are far apart. But when NPs come closer to each other, the additional hot spots emerge in the space between NPs, as well as those under the NPs. Thus, the Raman signals from any analytes that could get into the mentioned regions will get enhanced. Those effects have been demonstrated in Ref. [71]. Again, varying electrode potential altered the average distance between the NPs with their negatively charged ligands; more positive electrode polarisation created a denser array of NPs, and amplified the SERS signal.

The Raman signal from the analytes was increasing proportionally to the increase of the number of NPs on the surface but only when NPs were on average still far from each other. Further densification of NP array was making this dependence nonlinear, thus demonstrating clear emergence of hot spots not only underneath NPs but also in between NPs, when they come closer to each other, and, plasmon resonance wise, start 'talking with each other'! In the same work [71], measurements of the Raman signals were paralleled by simple reflectivity measurements. The latter allowed to retrieve the average distances between NPs, control the proximity of the measured and calculated reflections spectra (with full success, again), and with thus evaluated distances between NPs in the monolayer to calculate the enhancement of Raman intensity.

Note that the idea of building metasurface for enhanced Raman signal by designed arrays of plasmonic nano-objects has long history [read, e.g. 72-74], although the funky term 'metasurfaces' was not used then. What was shown in Ref. 71 is that one can manipulate the structure of such arrays, 'electroadsorbing'/'electrodesorbing' them onto/from solid electrodes, at our will and in real time, and how it thereby affects the SERS signal, demonstrating a crucial role of electromagnetic enhancement mechanism [75].

Correspondence between theory and experiments was perfect.

Few other configurations have been considered, based on similar principles.

This is, e.g. a theory of functioning of Fabry-Perot cell (cavity) that would 'trap' light between two semi-transparent electrode plates, and filter the transmission of light through the cell, by controlling the density of NP arrays on the plates through NP electrosorbtion/desorption from/to electrolyte solution filling the cavity [76, 77]. It is an interesting alternative to the standard mechanical adjustment of the cavity length, as it shows opportunities of sharp light transmission filtering by tiny voltage variation.

Another example goes third dimension: electro-feeding with NPs of the void space between transparent electronically conducting $\mathrm{ZnO}$ nano-columns, subject to the applied voltage [78]. The electrodeposition of metals into well-defined porous structures and dimensions is well-known, extending as far as to growing ultrathin nanowires in metal-organic frameworks 
(MOFs) that can function as versatile templates for the growth of metallic nanostructures with precisely controlled shapes and sizes (see e.g. [79, 80]). We know how to electrosorb large ionic liquid ions into MOF pores [81]. But, to my knowledge, building columns of individual NPs by physical electrodeposition in pores of large size has not yet been tried.

Interesting opportunity could be offered by anchored systems: changing orientation with respect to the electrode of the metallic (plasmonic) 'flat' nano-cuboids linked on one narrow edge to a transparent electrode, with variation of electrode polarisation. The calculation shows a remarkable effect of the reorientation of cuboids with respect to the electrode on the overall optical response of the interface [82], with fast dynamics, as not depending on translational diffusion but only reorientation of cuboids.

None of these three last examples have been yet experimentally tested, so they remain hypothetic systems. But all the previous experience with their sister systems suggests that they would work as predicted by the theory.

It was furthermore shown that interfacial optical properties of NP arrays can also be controlled not through applied electrode potentials, but temperature variation in a system that keeps the array together by thermo-auxetic ligands. Such thermally controlled optical switch was proposed by Joshua Edel. It was based on the self-assembly of poly(N-isopropyl-acrylamide)-functionalized gold nanoparticles on a planar macroscale gold substrate, as been built and explored by Ye Ma, Deb Sikdar, et al in Ref. [83] Shrinking of these ligands with temperature increase and expansion with temperature decrease affects inter-NPs separation; this effect can be calibrated theoretically and experimentally, in a similar way as in electrosorptioncontrolled arrays. Again, this particular system was shown also to operate in harmony with the theory [83].

One should also mention a series of works by the Dryfe's group in Manchester related to different aspects of localisation of NPs at ITIES, including voltage control over the density of adsorbed NP arrays. For instance, they have published a paper on electrotuning SERS at the ITIES by voltage-controlled population of silver NPs at the interface [84]. I will not go into any further details of the works of this group, referring the reader to a comprehensive review article by Booth and Dryfe [85].

There were a number of works exploring electrocatalytic properties of self-assembled layers of NPs at ITIES, such as reported, e.g. in Ref. [86-88] . This subject is very interesting itself, but it lies beyond the central theme of this article; moreover, intriguing from the fundamental point of view, it is not clear yet how practical such systems could be. Indeed, liquid/ liquid interfaces are flat, but not volume-filling as required for industrial applications of electrocatalysis. Creating, however, decorated novel electrode materials by controlled selfassembly at such interfaces is an interesting direction to mention [89]. Preparation of solid-state nanomaterials using electrodeposition of larger size colloidal particles onto templated electrodes in ordinary electrochemical cells is a separate direction for electrochemical nanotechnology [90].

All in all, the thus far obtained results have demonstrated that the physical picture of the effects of electrochemical plasmonics has been properly understood, and it can be laid into basis of the corresponding electrochemically controlled optical devices, to be built resting on the obtained fundamental knowledge. As mentioned, this will require miniaturisation of electrochemical cells, moving to micro-cells, and microcompartmentalised cells. This is a long road. It will take time, efforts and money, to demonstrate the proof of concept of these novel devices. But the general direction has been made clear.

Now, how about electrochemical photonics, i.e. the effects beyond simple plasmonic resonances, such as reflectivity, SERS, and alike? I have listed the main directions of photonics above, many of which are based on much more sophisticated theories and experimental setups. But what electrochemistry could do is to make them electrotunable. This will refer preferably to super structures with the characteristic dimensions larger than wavelength of light.

A simple idea, proposed to us by one of the photonics champions - N. Zheludev, is to lay on the surface of an electrode (transparent or reflecting one, subject to the task of interest), an optically and electrochemically inert mask with any figures of interest cut out of the mask, thereby opening those sections to electrosorption of NPs. The population of those sections with NPs can then be controlled by polarising the electrode, leading to the variation of the corresponding effects of the optical properties of those sections. The underlying electrode could further be compartmentalised, so you could control the properties of each section independently. Options here are unlimited, subject to the researchers' imagination, ideally working in cooperation with cutting-edge gurus from the corresponding branches of photonics.

Electrochemistry is an amazing interdisciplinary science that had already 'donated its blood' to many branches of sciences and engineering, from energy storage to biology and medicine. I have outlined above a new, emerging avenue for it. But let's be honest, solid-state scientists and engineers (and photonics is based on solid-state physics and electronics) are afraid of anything that is 'wet'. Would 'wet photonics' ever take off? For fundamental investigations - why not, but for practical applications?

One way (absolutely not tried and not tested yet) is to use ionic liquids as electrolytes, perhaps slightly doped with organic solvent to reduce their viscosity (the so called "solventin-salt" systems). They are not volatile, behave almost like gels, even more than the aqueous electrolyte in the Dead Sea, and most of them are optically transparent. The question is whether they could let NPs move easily and fast away from or to the surface, and generally form dense electrosorbed monolayers at the interface. If they do, when implemented in micro-engineered electrochemical cells, the latter would not 
look wet at all, but quite fancy. But all this is got to be tried, and not knowing the result in advance, make it even more interesting!

Acknowledgements I am thankful to Joshua Edel, Anthony Kucernak, Debabrata Sikdar, Ye Ma, Cristian Zagar, Yunuen Montelongo, Eleanora Velleman, Michael Urbakh, Michael Flatte, and my other colleagues with whom I had a privilege and pleasure to work on electrochemical plasmonics, as well as leaders of other groups working in this area, in particular David Schiffrin, Hubert Girault, Mark Schlossman, and Rob Dryfe, for many useful discussions. Conversations with John Pendry, Nikolay Zheludev, Anatoly Zayats, and Tal Ellenbogen are greatly appreciated. I am thankful to Fritz Scholz for an invitation to write these notes in the special issue containing an introduction of new avenues in electrochemistry.

Open Access This article is licensed under a Creative Commons Attribution 4.0 International License, which permits use, sharing, adaptation, distribution and reproduction in any medium or format, as long as you give appropriate credit to the original author(s) and the source, provide a link to the Creative Commons licence, and indicate if changes were made. The images or other third party material in this article are included in the article's Creative Commons licence, unless indicated otherwise in a credit line to the material. If material is not included in the article's Creative Commons licence and your intended use is not permitted by statutory regulation or exceeds the permitted use, you will need to obtain permission directly from the copyright holder. To view a copy of this licence, visit http://creativecommons.org/licenses/by/4.0/.

\section{References}

1. Zheludev NI (2010) The road ahead for metamaterials. Science 328(5978):582-583

2. Li J-M (2016) Principles of photonics. Cambridge University Press, Cambridge

3. Reider GA (2016) Photonics, Springer

4. Banerjee S, Tyagi AK (eds) (2012) Functional Materials. Elsevier. https://doi.org/10.1016/C2010-0-65659-8

5. Tiwari A, Uzun L (eds) (2015) Adv Functional Mater. Scrivener Pbls LLC. https://doi.org/10.1002/9781118998977

6. Shahinpoor M (ed) (2020) Fundamentals of Smart Materials. RSC ISBN, 978-1-78262-645-9

7. Goodwin ZAH, Eikerling M, Lowen H, Kornyshev AA (2018) Theory of microstructured polymer-electrolyte artificial muscles. Smart Mater Struct 27:\#075056

8. Pillatsch P, Yeatman EM, Holmes AS (2012) A scalable piezoelectric impulse-excited energy harvester for human body excitation. Smart Mater Struct 21:\#115018

9. Pozzi M, Zhu M (2011) Plucked piezoelectric bimorphs for kneejoint energy harvesting: modelling and experimental validation. Smart Mater Struct 20:55007

10. Hsu T-H, Taylor JA, Krupenkin TN (2017) Energy harvesting from aperiodic low-frequency motion using reverse electrowetting. Faraday Discuss 199:377-392

11. Morrisey TG, Mitchell TG, Jaros AT, Ambos E, Keplinger C (2019) Mechanical-to-electrical energy conversion with variable electric double layers. Energy Technol 7:\#1801007

12. Kornyshev AA, Twidale R, Kolomeisky AB (2017) Current generating 'double layer shoe' with a porous sole: ion transport matters. J Phys Chem C 121:7583-7595
13. Wu C, Wang AC, Ding W, Guo H, Wang ZL (2019) Triboelectric nanogenerator: a foundation of the energy for the new era. Adv Energy Mater 9:\#1802906

14. Wang S, Lin L, Wang ZL (2012) Nanoscale triboelectric-effectenabled energy conversion for sustainably powering portable electronics. Nano Lett 12:6339-6346

15. Zhang J, Macdonals KF, Zheludev NI (2012) Contolling light-withlight without nonlinearity. Light Sci Appl 1:e18

16. Zheludev NI, Plum E (2016) Reconfigurable nanomechanical photonic metamaterials. Nat Nanotech 11:16-22

17. Yi C, Si KJ, Sikdar D, Tang Y, Premaratne M, Cheng W (2015) Ultrathin plasmene nanosheets as soft and surface-attachable sers substrates with high signal uniformity. Adv Opt Mater 3:919-924

18. Zhu W, Xiao F, Kang M, Sikdar D, Premaratne M (2014) Tunable terahertz left-handed metamaterial based on multi-layer graphenedielectric composite. Appl Phys Lett 104:\#051902

19. Xiong W, Sikdar D, Walsh M, Si KJ, Tang Y, Chen Y, Mazid R, Weyland M (2013) Single-crystal caged gold nanorods with tunable broadband plasmon resonances. Chem Commun 49:9630 9632

20. Guo P, Sikdar D, Huang X, Si KJ, Su B, Chen Y, Xiong W, Yap LW, Premaratne M, Cheng W (2014) Large-scale self-assembly and stretch-induced plasmonic properties of core-shell metal nanoparticle superlattice sheets. J Phys Chem C 118:26816-26824

21. Bethell D, Schiffrin DJ, Kiely C, Brust M, Fink J (2001) Selfassembly of nanostructured materials: dreams and reality. In: Hyper-Structured Molecules II : Chemistry, Physics and Applications, pp 179-195

22. Flatte ME, Kornyshev AA, Urbakh M (2010) Electrovariable nanoplasmonics and self-assembling smart mirrors. J Phys Chem C 114:1735-1747

23. Maier SA (2007) Plasmonics: Fundamentals and applications. Springer, New York

24. Otto A (1968) Excitation of nonradiative surface plasma waves in silver by the method of frustrated total reflection. Z f Physik 216: 398-410

25. Kretschmann E, Raether H (1968) Radiative decay of non-radiative surface plasmons excited by light. Z Naturforsch 23A:2135

26. Raether H (1988) Surface plasmons on smooth and rough surfaces and on gratingsr. Springer-Verlag

27. Pines D, Bohm D (1952) A collective description of electron interactions: II collective vs individual particle aspects of interactions. Phys.Rev. 85:338-352

28. Richie R (1957) Plasma losses by fast electrons in thin films. Phys Rev 106:874-881

29. Fleischmann M, Hendra PJ, McQillain AJ (1974) Raman spectra of pyridine adsorbed at silver. Chem Phys Lett 26:163-166

30. Jeanmaire DL, Van Duyne RP (1977) Surface Raman spectroelectrochemistry. J Electroanal Chem 84:1-20

31. Willets KA, Van Duyne RP (2007) Localized surface plasmon resonance spectroscopy and sensing. Annu Rev Phys Chem 58:267297

32. Stiles PL, Dieringer JA, Shah NC, Van Duyne RP (2008) Surfaceenhanced Raman spectroscopy. Annu Rev Anal Chem 1:601-626

33. (2006) See chapters. In: Kneipp K, Moskovits M, Kneipp H (eds) Surface-enhanced Raman scattering. Topics in Applied Physics, 103. Springer, Berlin, Heidelberg

34. Albrecht MG, Creighton JA (1977) Anomalously intense Raman spectra of pyridine at a silver electrode. J Am Chem Soc 99:52155217

35. Otto A, Futamata M (2006) Electronic mechanisms of SERS. In: Kneipp K, Moskovits M, Kneipp H (eds) Surface-enhanced Raman scattering. Topics in Appl. Phys, vol 103. Springer, Berlin, Heidelberg, pp 147-182

36. Brodsky MA, Urbakh M (1990) Optical properties of microrough metal surfaces. Prog Surf Sci 33:91-170 
37. Chao F, Costa M, Tadjeddine A (1992) The use of optics for understanding the electrochemical interface. J Electroanal Chem 329: 313-327

38. Zheludev NI, Kivshar YI (2012) From metamaterials to metadevices. Nat Mater 11:917-924

39. Lustenberger P, Rohrer H, Christoph R, Siegenthaler H (1988) Scanning tunneling microscopy at potential controlled electrode surfaces in electrolytic environment. J Electroanal Chem 243: 225-235

40. Christoph R, Siegenthaler H, Rohrer H, Wiese H (1989) In situ scanning tunneling microscopy at potential controlled $\mathrm{Ag}(100)$ substrates. Electrochim Acta 34:1011-1022

41. Binning G, Rohrer H, Gerber C, Wiebel E (1982) Surface studies by scanning tunneling microscopy. Phys Rev Lett 49:57-61

42. Binning G, Rohrer H (1982) Scanning tunneling microscopy. Helv Phys Acta 55:726-735

43. Simon P, Gogotsi Y (2008) Materials for electrochemical capacitance. Nat Mater 7:845-854

44. Kasani S, Curtin K, Wy N (2019) A review of 2D and 3D plasmonin nanostructure array patterns: fabrication, light management, and sensing applications. Nanophotonics 8:2065-2089. https://doi.org/10.1515/nanoph-2019-0158

45. Huang FM, Wilding D, Speed JD, Russell AE, Bartlett PN, Baumberg JJ (2011) Dressing plasmons in particle-in-cavity architectures. Nano Lett 11:1221-1226

46. Mahajan S, Cole RM, Soares BF, Pelfrey SH, Russell AE, Baumberg JJ, Bartlett PN (2009) Relating SERS intensity to specific plasmon modes on sphere segment void surfaces. J Phys Chem C 113:9284-9289. https://doi.org/10.1021/jp900661u

47. Cheng Y, Schiffrin DJ (1996) Electrodeposition of metallic gold clusters at the water/1,2-dichloroethane interface. J Chem Soc Faraday Trans 92:3865-3871

48. Sendroiu IE, Mertens SFL, Schiffrin DJ (2006) Plasmon interactions between gold nanoparticles in aqueous solution with controlled spatial separation. Phys Chem Chem Phys 8:1430-1436

49. Schiffrin D (2015) Nanoparticles and self-organisation: the emergence of hierarchical properties from the nanoparticle soup (i.e., the small is getting bigger). Faraday Discuss 181:481-487

50. Antoine R, Brevet PF, Girault HH, Bethell D, Schiffrin DJ (1991) Surface plasmon enhanced non-linear optical response of gold nanoparticles at the air/toluene interface. Chem Comm 19:19011902

51. Su B, Abid JP, Fermin DJ, Girault HH, Hoffmannova H, Krtil P, Samec Z (2004) Reversible voltage-induced assembly of Au nanoparticles at liquid vertical bar liquid interfaces. J Am Chem Soc 126:915-919

52. Girault HH, Schiffrin DJ (1989) Electrochemistry of liquid-liquid interfaces. Electroanal Chem 15:1-141

53. Girault HH (2010) In: Bard AJ, Zoski CG (eds) Electroanal. Chem. CRC Press, Boca Raton, pp 1-104

54. Sikdar D, Kornyshev AA (2016) Theory of tailorable optical response of two dimensional arrays of plasmonic nanoparticles at dielectric interfaces. Sci Rep 6:\#33712

55. Sikdar D, Bin Hasan S, Urbakh M, Edel J, Kornyshev AA (2016) Unravelling the optical responses of nanoplasmonic mirror-onmirror metamaterials. Phys.Chem.Chem.Phys. 18:20486-20498

56. Edel J, Kornyshev AA, Kucernak A, Urbakh M (2016) Fundamentals and applications of self-assembled plasmonic nanoparticles at interfaces. Chem Soc Rev 45:1581-1596

57. Turek VA, Cecchini MP, Paget J, Kucernak AR, Kornyshev AA, Edel JB (2012) Plasmonic ruler at liquid-liquid interface. ACS Nano 9:7789-7799

58. Yang ZL, Chen S, Fang PP, Ren B, Girault HH, Tian ZQ (2013) LSPR properties of metal nanoparticles adsorbed at a liquid-liquid interface. PhysChemChemPhys 15:5374-5378
59. Cecchini MP, Turek VA, Paget J, Kornyshev AA, Edel JB (2013) Self-assembled nanoparticle arrays for multi-phase trace analyte detection. Nat Mater 12:165-171

60. Bera MK, Chan H, Moyano DF, Yu H, Tatur S, Amoanu D, Bu W, Rotello VM, Meron M, Kral P, Lin B, Schlossman ML (2014) Interfacial localization and voltage-tunable arrays of charged nanoparticles. Nano Lett 14:6816-6822

61. Velleman L, Sikdar D, Turek VA, Kucernak AR, Roser SJ, Kornyshev AA, Edel J (2016) Tuneable 2D assembly of plasmonic nanoparticles at liquid|liquid interfaces. Nanoscale 8:19229-19241

62. Fang PP, Chen S, Deng HQ, Scanlon MD, Gumy F, Lee HJ, Momotenko D, Amstutz V, Cortes-Salazar F, Pereira CM, Yang ZL, Girault HH (2013) Conductive gold nanoparticle mirrors at liquid/liquid interfaces. ACS Nano 7:9241-9248

63. Montelongo Y, Sikdar D, Ma Y, McIntosh AJS, Velleman L, Kucernak AR, Edel JB, Kornyshev AA (2017) Electrotuneable nanoplasmonic liquid mirror. Nat Mater 16:1127-1135

64. Ramos A, Garcia-Sanchez P, Morgan H (2016) AC electrokinetics of conducting microparticles: a review. Curr Opin Colloid Interface Sci 24:79-90

65. Ramos A, Morgan H, Green NG, Castellanos A (1998) AC electrokinetics: a review of forces in microelectrode structures. J Phys D Appl Phys 31:2338-2235

66. Kornyshev AA, Marinescu M, Paget J, Urbakh M (2012) Reflection of light by metal nanoparticles at electrodes. PhysChemChemPhys 14:1850-1859

67. Truong V-V, de Dormale B (2011) Optical absorption in overcoats of nanoparticle arrays on a metallic substrate. Plasmonics 6:195200

68. Mock JJ, Hill RT, Tsai Y-J, Chilkoti A, Smith DR (2012) Probing dynamically tunable localized surface plasmon resonances of filmcoupled nanoparticles by evanescent wave excitation. Nano Lett 12: $1757-1764$

69. Baum T, Bethell D, Brust M, Schiffrin DJ (1999) Electrochemical charge injection into immobilized nanosized gold particle ensembles: potential modulated transmission and reflectance spectroscopy. Langmuir 15:866-871

70. Ma Y, Zagar C, Klemme DJ, Sikdar D, Velleman L, Montelongo Y, Oh SH, Kucernak AR, Edel JB, Kornyshev AA (2018) Tunable nanoplasmonic mirror at an electrochemical interface. ACS Photonics 5:4604-4616

71. Ma Y, Sikdar D, Fedosyuk A, Velleman L, Klemme DJ, Oh SH, Kucernak AR, Kornyshev AA, Edel JB (2020) Electrotunable nanoplasmonics for amplified surface enhanced Raman spectroscopy. ACS Nano 14:328-336

72. Tian ZQ, Ren B, Wu DY (2002) Surface enhanced Raman Scattering; from noble to transition metals and from rough surfaces to ordered structures. J Phys Chem B 106:9463-9483

73. Tian ZQ (2005) Surface enhanced Raman spectroscopy: advancement and applications. J. Raman Spectroscopy 36:466-470

74. Li JF, Huang YF, Ding Y, Yang ZL, Li SB, Zhou XS, Fan FR, Zhang W, Zhou ZY, Wu DY, Ren B, Wang ZL, Tian ZQ (2010) Shell-isolated nanoparticle-enhanced Raman spectroscopy. Nature 464:392-395

75. Ding SY, You EM, Tian ZQ, and Moskowitz M (2017) Electromagnetic theories of surface enhanced Raman scattering. Chem Soc Rev 46:4042-4076

76. Weir H, Edel JB, Kornyshev AA, Sikdar D (2018) Towards electrotuneable nanoplasmonic Fabry-Perot Interferometer. Sci Rep 8. https://doi.org/10.1038/s41598-017-19011-4

77. Sikdar D, Kornyshev AA (2019) An electro-tunable Fabry-Perot interferometer based on dual mirror-on-mirror nanoplasmonic metamaterials. Nanophotonics 8:2279-2290

78. Sikdar D, Hayley H, Kornyshev AA (2019) Optical response of electro-tuneable 3D superstructures of self-assembling plasmonic on transparent columnar electrodes. Opt Express 27:26483-26498 
79. Volosskiy B, Niwa K, Chen Y, Zhao Z, Weiss NO, Zhong X, Ding M, Lee C, Huang Y, Duan X (2015) Metal-organic framework templated synthesis of ultrathin, well-aligned metallic nanowires. ACS Nano 9:3044-3049

80. Worrall SD, Bissett MA, Hill PI, Rooney AP, Haigh SJ, Attfield MP, Dryfe RAW (2016) Metal-organic framework templated electrodeposition of functional gold nanostructures. Electrochim Acta 222:361-369

81. Bi S, Banda H, Chen M, Niu L, Chen M, Wu T, Wang J, Wang RX, Feng JM, Chen T, Dinca M, Kornyshev AA, Feng G (2020) Molecular understanding of charge storage and charging dynamics in supercapacitors with MOF electrodes and ionic liquid electrolytes. Nat Mater 19:552-558

82. Sikdar D, Bucher AO, Zagar C, Kornyshev AA (2017) Electrochemical plasmonic metamaterials: towards fast electrotuneable reflecting nanoshutters. Faraday Discuss 199: 585-602

83. Ma Y, Sikdar D, Fedosyuk A, Velleman L, Zhao MG, Tang LH, Kornyshev AA, Edel JB (2019) Auxetic thermoresponsive nanoplasmonic optical switch. ACS Appl Mater Interfaces 11: 22754-22760

84. Booth SG, Cowcher DP, Goodacre R, Dryfe RAW (2014) Electrochemical modulation of SERS at the liquid/liquid interface. Chem Commun 50:4482-4484
85. Booth SG, Dryfe RAW (2015) Assembly of nanoscale objects at the liquid/liquid Interface J. Phys Chem C 119:23295-23309

86. Grunder Y, Fabian MD, Booth SG, Plana D, Fermin DJ, Hill PI, Dryfe RAW (2013) Solids at the liquid-liquid interface: electrocatalysis with pre-formed nanoparticles. Electrochim Acta 110:809-815

87. Scanlon MD, Peljo P, Mendez MA, Smirnov E, Girault HH (2015) Charging and discharging at the nanoscale: Fermi level equilibration of metallic nanoparticles. Chem Sci 6:2705-2720

88. Trojanek A, Langmaier J, Samec Z (2006) Electrocatalysis of the oxygen reduction at a polarised interface between two immiscible electrolyte solutions by electrochemically generated Pt particles. Electrochem Commun 8:475-481

89. Ananthoju B, Biroju RK, Theis W, Dryfe RAW (2019) Controlled electrodeposition of gold on graphene: maximization of the enhanced Raman scattering response. Small 15:\#1901555

90. Abdelsalam ME, Bartlett PN, Baumberg JJ, Coyle S (2004) Preparation of arrays of isolated spherical cavities by selfassembly of polysterene spheres on self-assembled pre-patterned macroporous films. Adv Mater 16:90-93

Publisher's note Springer Nature remains neutral with regard to jurisdictional claims in published maps and institutional affiliations. 